International Journal of Medical Sciences

ISSN 1449-1907 www.medsci.org 2007 4(5):247-248

Letter to the editor

CIvyspring International Publisher. All rights reserved

\title{
Comment on Childhood Febrile Seizures: Overview and Implications by Tonia Jones and Steven J. Jacobsen
}

\author{
Efterpi Pavlidou and Christos P. Panteliadis \\ Paediatric Department, Aristotle University of Thessaloniki, Thessaloniki, Greece
}

Correspondence to: Dr. Christos P. Panteliadis, Professor of Paediatric Neurology, Aristotle University of Thessaloniki, Vas. Olgas 97, Thessaloniki, Greece, Tel-Fax: ++302310 854429. Email cpantel@hol.gr

Received: 2007.09.05; Accepted: 2007.10.03; Published: 2007.10.15

Reading the review article "Childhood Febrile Seizures: Overview and Implications" by Jones and Jacobsen that was recently published in International Journal of Medical Sciences (Int J Med Sci 2007; 4:110-114, http://www.medsci.org/v04p0110.htm), we noticed some points which we believe are worth mentioning. Additionally, we would like to report our own data from a large prospective study with a long-term follow up.

The authors make a brief reference to the predictors of febrile seizure (FS) recurrence; however they do not distinguish between the first recurrence and further ones. In our recently published study we identified not only the prognostic factors for the first recurrence but also for further ones. Statistical analysis showed as significant prognostic factors for the first recurrence of FS: low age at onset (<18 months), low duration of fever ( $<12$ hours), height of fever up to $38.5^{\circ} \mathrm{C}$, recurrence within the same febrile illness, neurological abnormalities, family history of FS and particularly maternal preponderance. The latter one is an extremely strong prognostic marker, for the first time mentioned in literature. Prognostic factors for the second or further recurrences revealed that the factors retaining their prognostic value were: family history of FS and low age at onset. The rest factors lost their power for the prognosis of multiple recurrences [3].

As far as the issue of management of FS is concerned, intermittent rectal diazepam prophylaxis is found in our study to be effective and safe for the prevention of FS recurrence only in high-risk population [4]. Others have evaluated the use of oral diazepam prophylaxis at times of fever and demonstrated significant reduction in the percentage of febrile seizure recurrence $[5,6]$ who also report minor and reversible side effects. Many are concerned that the side effects of diazepam may delay the diagnosis of an underlying illness (e.g. meningitis or encephalitis). Infection of central nervous system must always be considered in case of a seizure with fever, especially in infants less than 18 months of age. When the clinician is uncertain, a lumbar puncture should be performed. In our opinion, parental education is essential and optimal management of febrile seizures involves preventing their recurrence only in those populations that are at high risk. This can be done most effectively with intermittent rectal diazepam provided that it is given at the onset of fever.

The authors do not refer at all to the prognostic factors for the subsequent development of epilepsy after FS, which would have been a valuable additional parameter to be included in their review article. Major risk factors for the occurrence of epilepsy after FS are: age at onset of FS older than 3 years old, family history of epilepsy, complex FS and principally multiple episodes of FS (more than four). Rational thought is that since multiple febrile seizures lead to epilepsy and short duration seizures beget new seizures [1, 2], then the prevention of febrile seizures may reduce the risk of subsequent epilepsy. However, studies have shown no difference in the occurrence of epilepsy among children who received prophylaxis for FS and those without prophylaxis. It is possible that a common genetic predisposition is responsible for the development of epilepsy after FS, rather than an underlying lesion caused by recurrent seizures. However, large prospective studies are needed in order to verify that prevention of FS does not also prevent subsequent epilepsy.

\section{Conflict of interest}

The authors have declared that no conflict of interest exists.

\section{References}

1. Berg AT and Shinnar S. Unprovoked seizures in children with febrile seizures: short-term outcome. Neurology 1996;47:562-568

2. Berg AT and Shlomo S. Do seizures beget seizures? An assessment of the clinical evidence in humans. Journal of Clinical Neurophysiology 1997;14: 102-110

3. Pavlidou E, Tzitiridou M, Kontopoulos E, Panteliadis C. Which factors determine febrile seizure recurrence? A prospective study. Brain and Development 2007; [Epub ahead of print]

4. Pavlidou E, Tzitiridou M, Panteliadis C. Effectiveness of intermittent rectal diazepam prophylaxis in febrile seizures: A long term prospective study. J of Child Neurology 2006; 21: 
1036-1040

5. Rosman NP, Colton T, Labazzo J, et al. A controlled trial of diazepam administered during febrile illnesses to prevent recurrence of febrile seizures. N Engl J Med 1993; 329:79-84

6. Verrotti A, Latini G, Di Corcia G, et al. Intermittent oral diazepam prophylaxis in febrile convulsions: Its effectiveness for febrile seizure recurrence. Eur J of Pediatr Neurol 2004; 8:131134 